\title{
Optical Changes in Rock-salt CdS System under Increasing Silver Concentrations Effects
}

\author{
M. Junaid Iqbal Khan ${ }^{a, *}$, Zarfishan Kanwal ${ }^{a}$, M. Nauman Usmani ${ }^{a}$, \\ PerveEn AkhtaR $^{a}$, AzeEm Nabi $^{b}$ And Nisar Ahmad ${ }^{c}$ \\ ${ }^{a}$ Laboratory of Theoretical and Experimental Physics, Department of Physics, Bahauddin Zakariya University, \\ Multan, (60800), Pakistan \\ ${ }^{b}$ Department of Physics, University of Gujrat, Gujrat, Pakistan \\ ${ }^{c}$ Department of Physics and Applied Mathematics, Pakistan Institute of Engineering and Applied Sciences, \\ Islamabad, Pakistan
}

(Received November 27, 2017; in final form December 19, 2018)

\begin{abstract}
Current research is a computational study on optical properties of Ag doped CdS based on the PerdewBurke-Ernzerhof parameterized generalized gradient approximations. Observing change in optical properties under various concentrations on CdS:Ag system, by fixing supercell size is a focus of study using density functional theory. Computational formulation is done by substitution of $\mathrm{Cd}$ atoms with $\mathrm{Ag}$ atoms in host CdS lattice. Results reveal that optical properties are enhanced upon increasing dopant concentration. Optical absorption shows blueshift and increases in visible region. Coupling of $\mathrm{S} p$ and $\mathrm{Ag} s$ states are obvious from plots of density of states. However, $\mathrm{Ag}$ doping brings significant change in optical properties which may lead towards advocacy of CdS for optoelectronic devices.
\end{abstract}

DOI: 10.12693/APhysPolA.134.1099

PACS/topics: cadmium sulfide, Ag-doping, DFT calculations, density of states, optical properties

\section{Introduction}

During past decades, numerous research studies have been conducted in order to understand optical properties of certain semiconductors. It helps in fabricating devices and reshaping technology at nanoscale. Nanomaterials due to their quantum effects and opto-magnetic properties have tremendous potential applications in optoelectronics and spintronics [1,2]. Few of semiconductors including CdSe, ZnS, ZnSe and CdS have been studied at nanoscale $[3,4]$. Cadmium sulfide (CdS) is most studied material among II-VI group semiconductors due to its exceptional optical and magnetic properties especially owing to its band gap $2.42 \mathrm{eV} \mathrm{[5].} \mathrm{It} \mathrm{has} \mathrm{striking} \mathrm{im-}$ pact in revolutionizing areas of research related to solar cells [6-8], photocatalysis [9, 10], photodetectors [11, 12], biotechnology [13], field effect transistors, and in certain gas detectors. Moreover, it is still proving itself as best candidate for areas of spintronics, non-linear optics [14], light dependent resistors [15], and pigment solar cells [16]. CdS has also been widely used in study of detecting visible light.

CdS has three different structural occurrences in nature which are wurtzite (WZ), zinc blende (ZB), and rock salt. They are identified on basis of certain parameters called lattice constants. Hexagonal symmetry of zinc-blende structures is based on four atomic basis

\footnotetext{
* corresponding author; e-mail drjunaid.iqbalkhan@bzu.edu.pk
}

and exhibits polymorphous behavior. Wurtzite [17] and zinc-blende structures of CdS are degenerated energetically. Formation of wurtzite structure is face centered cubic lattice while zinc-blende structure is hexagonal. Rock-salt structure of CdS exists in cubic form which is generally found in nanocrystallite form [18, 19], emphasizing its uses in recent development of nanotechnology. Due to nanotechnological applications of rock salt CdS, it is mostly studied in research for predicting electronic, electrical and optical properties. Because of these reasons, rock-salt phase of CdS is preferred over WZ and ZB phases. Junaid Iqbal Khan et al. [20] have theoretically worked with rock-salt $\mathrm{CdS}$ in order to find optical properties with $\mathrm{Cu}$ doping. Current research work (CdS:Ag) shows interesting results which have been first ever reported with strong literature background. All results have been expressed graphically and show good comparison of pure $\mathrm{CdS}$ with varying concentration of dopant $(\mathrm{Ag})$. Hence this study is unique and alluring in its nature for readers.

Over past years, much of research on CdS as host material has been conducted experimentally but theoretical study particularly focused on optical properties are hardly found. Doping CdS with certain dopants is expected to enhance optical, electrical, and electronic properties. A number of experimentalists have conducted research in order to investigate physical properties of doped CdS [21-24]. Theoretical studies which mandate experimental outcomes of CdS are not developed. Doping transition metals with CdS have revealed interesting facts which further emphasizes its uses in most of recent technological applications [25-27]. Ristova et al. 
have experimentally studied Ag doped CdS thin films system by chemical deposition through ion exchange process. Their findings consist of X-ray diffraction (XRD) spectrum, increase of dark conductivity and decrease of photoconductivity upon increasing Ag contents. The increase in conductivity signifies its window layer application in photovoltaic cells [28]. Saikia et al. have carried out experimental study of CdS quantum dots which were prepared in thin film by dip coating technique and their study constituted by XRD, optical properties (absorption and transmission), scanning electron microscopy (SEM) and transmission electron microscopy (TEM) analysis. Through their experimental analysis, they reported that by doping silver into CdS, solar efficiency is increased [29]. Also, Grodzyuk et al. have done experimental study of $\mathrm{Ag}$ doped $\mathrm{CdS}$ nanoparticles and found that silver doping in CdS may bring an increase in the luminescence intensity by five times. They concluded that addition of $\mathrm{Ag}$ ions in $\mathrm{CdS}$ nanoparticles will not bring any remarkable change in nanocrystal [30]. Mochahari et al. in 2017 [31] have observed increase in dielectric constant and refractive index which increases with increasing ion fluence. All experimental studies have been focused on nanoparticles of CdS [4] but theoretical study has not been presented yet which will demonstrate comparison of experimental and theoretical results. Jianbo Yin et al. have done experimental and theory simulation of Ag substituted Cd in CdS quantum dots. They found change in optical properties due to Ag substitution [32]. Their theoretical results are achieved using Cambridge Serial Total Energy package module (CASTEP) of material studio 6.0 version. Theoretical findings related to rock-salt CdS:Ag system which may be compared with experimental results are rarely found in literature and it is motivation of our study. Hence theoretical study of $\mathrm{Ag}$ doped CdS is found in literature using CASTEP but results obtained by Wien $2 \mathrm{k}$ code are for the first time reported in our study.

In the present research work, we focus on computational approach while doping $\mathrm{Ag}$ into $\mathrm{CdS}$ host lattice. We shall calculate optical properties including absorption, reflectance, dielectric constant, index of refraction, extinction coefficient and conductivity by substituting $\mathrm{Cd}$ with $\mathrm{Ag}$ atoms. Partial density of states (PDOS) and total density of states (TDOS) of doped system are to be calculated. The Perdew-Burke-Ernzerhof (PBE) parameterized generalized gradient approximations (PBE-GGA) is employed using density functional theory (DFT). Results are compared with those reported in literature. Wien $2 \mathrm{k}$ code is used for entire calculations in order to meet challenges of computational field.

\section{Computational method}

In current study, we assumed rock-salt structure of CdS for calculations in order to find change in optical properties which may have surprising applications. A supercell of $1 \times 2 \times 2$ configuration with 32 atoms is simulated. We performed first principle calculations using PBE-GGA approximations and observed concentration effects on electronic and optical properties of $\mathrm{Ag}$ doped rock-salt CdS. PDOS, TDOS and optical properties have been calculated for Ag concentration of $3.12 \%$, $5.88 \%, 9.37 \%$, and $12.5 \%$ while substituting $\mathrm{Cd}$ atoms by $\mathrm{Ag}$ atoms in host $\mathrm{CdS}$ (rock salt) lattice. Impurity atoms $\left(\mathrm{Ag}^{+1}\right)$ were easily incorporated due to smaller ionic radius $(0.94 \AA)$ in comparison to $\mathrm{Cd}^{+2}$ atoms having larger radius $(0.97 \AA)$ [33]. Supercell is symmetrically simulated, based on $\alpha=\beta=\gamma=90^{\circ}$ and $F m-3 m$ space group. Atomic relaxations are executed by employing self-consistency criterion with minimum charge and energy convergence $10^{-2} \mathrm{C}$ and $10^{-2} \mathrm{eV}$, respectively. The Monkhorst-pack scheme was opted for $k$-points and all calculations are looped with $1000 k$-points sampling. Optical properties such as optical absorption, conductivity, extinction coefficient, reflectivity, index of refraction and dielectric constant have been evaluated through simulation of corresponding crystal geometries optimized by computational scheme. Moreover, comprehensive comparison of change in optical properties for case of pure $\mathrm{CdS}$ and for various dopant $(\mathrm{Ag})$ concentrations is presented. Several interesting facts have been explored in entire context with strong literature background.

\section{Results and discussions}

Structural forms of CdS exist in three configurations (wurtzite, zinc-blende, and rock-salt forms), among which the rock-salt form (cubic phase) is most famous due to its existence in nanocrystallites or nanoparticles. Also due to quantum confinement effects, these structures (rock-salt phases) carry importance. Figure 1 represents $1 \times 2 \times 2$ supercell images of rock-salt CdS structure for pure CdS and with $3.12 \%, 6.25 \%, 9.35 \%$, and $12.5 \% \mathrm{Ag}$ (dopant) concentrations at various configurations such as $(0,0,0)$, $[(0,0,0),(0,0,1 / 2)],[(0,0,0),(0,0,1 / 2),(0,1 / 2,0)],[(0,0,0)$, $(0,0,1 / 2),(0,1 / 2,0),(0,1 / 2,1 / 2)]$. Due to presence of im-

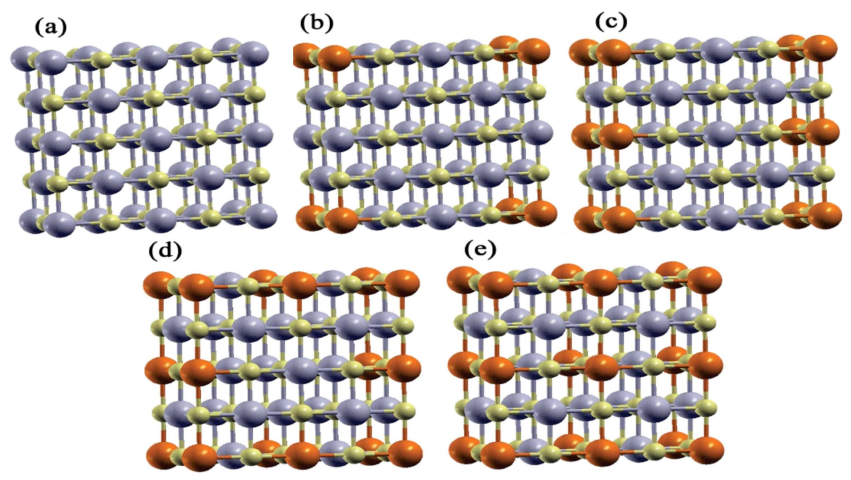

Fig. 1. Rock-salt structure of Ag doped CdS $(1 \times 2 \times 2)$ for, (a) pure CdS, (b) $3.12 \%$, (c) $5.88 \%$, (d) $9.37 \%$ and (e) $12.5 \% \mathrm{Ag}$-concentration effect. Blue, green, and red are $\mathrm{Cd}, \mathrm{S}$, and $\mathrm{Ag}$, respectively. 
purity $\mathrm{Ag}$ atoms at different lattice sites as well as increase of the number of impurity atoms, variations in the electronic and optical properties of the doped system are expected. This might be caused by the variations in the electronic configurations and change in the rearrangement and redistribution of local charges.

Possible information about occupying energy states of electrons can be encoded in context of DOS which provides detail of states to be occupied. Orbitals from individual atoms within a particular supercell configuration usually involve in composing DOS. TDOS and PDOS for pure and silver $(\mathrm{Ag})$ doped rock-salt $\mathrm{CdS}$ with various concentrations are demonstrated in Figs. 2-4 on energy axis. The Fermi level is shown in all plots by dotted line which shows separation of valence and conduction band. It is obvious that there is coupling of $\mathrm{Cd} s, \mathrm{~S} p$, and $\mathrm{Ag} s$ orbitals. Silver has one valence electron $\left(5 s^{1}\right)$ in atomic state which is isolated but upon doping its valence electrons can make bonds with neighboring sulfur atoms. Addition of $\mathrm{Ag}$ atoms (dopant) in CdS host lattice may provide enough number of electrons adjacent to the Fermi surface and this mechanism may be explained by DOS plots.
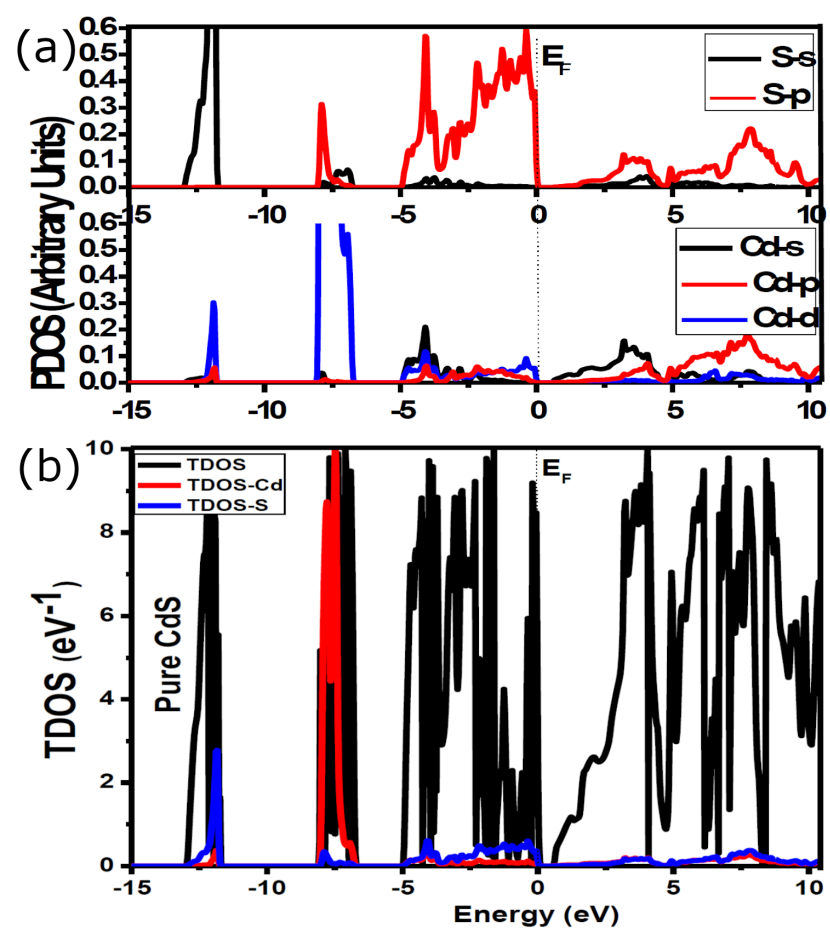

Fig. 2. (a) Partial density of states and (b) total density of states of pure CdS.

Present study is a computational work based on $\mathrm{Ag}$ doped rock-salt CdS system. The orbital trend for pure CdS $1 \times 2 \times 2$ supercell configuration has been demonstrated in Fig. 2 as TDOS and PDOS. It is clear that $\mathrm{Cd} 5 s$ and $\mathrm{S} 3 p$ make covalent bonds and electrons from these orbitals mutually form CdS compound. Two electrons are shared by $\mathrm{Cd}$ with that of $\mathrm{S}$ atom and as a result $\mathrm{S}^{2-}$ and $\mathrm{Cd}^{2+}$ are formed. From graphical trends of DOS for pure rock-salt CdS, it is clear that top of valence band $(0$ to $-5 \mathrm{eV})$ mainly comprises of $\mathrm{S} p$ and $\mathrm{Cd} d$ bonding states while conduction band ( 0 to $4.5 \mathrm{eV}$ ) contains bonding states of $\mathrm{Cd} s$ and $\mathrm{S} p$. It is observed that sulfur $p$-orbitals play prominent role in conduction process. Also, conduction band minimum has least impact of $\mathrm{Cd} d$ states and $\mathrm{S} s$ states.

From PDOS presented in Fig. 3, we can interpret electronic behavior of $\mathrm{Ag}$ doped $\mathrm{CdS}$ system with various concentrations. Figure 3 is representation of PDOS for all concentrations including $3.12 \%, 6.25 \%, 9.37 \%$, and $12.5 \%$. Various plots for specific concentration denote orbital coupling in both valence and conduction band. Transition of electrons in each concentration is remarkably observed. Individual states of each constituent atom have been figured in energy range -15 to $10.5 \mathrm{eV}$. These states show narrow and extending distribution in both transition bands. After heedful analysis of PDOS and TDOS, it is observed that individual states of $\mathrm{Ag}$ doped $\mathrm{CdS}$ system is contributed in three different regions. First region in valence band is restricted in range -5.3 to $-0.1 \mathrm{eV}$. In this region $\mathrm{Cd} s, \mathrm{~S} p$ and $\mathrm{Ag} d$ states are most prominent with least impact of $\mathrm{Ag} s$ and $\mathrm{S} s$ states. In third concentration $(9.37 \%)$, first region contains stretched overlapping of $\mathrm{Cd} s, p$ and $d$-orbitals and lies in -6.3 to $-0.1 \mathrm{eV}$. Second region lies in range -8.3 to $-6.7 \mathrm{eV}$ and contains contributing states of $\mathrm{Cd} d$, $\mathrm{S} p$ with minor $\mathrm{Ag} d$ states. In CdS:Ag (9.37\%) concentration $\mathrm{Cd} d$ states are stretched significantly $(-9.1$ to $-7.7 \mathrm{eV}$ ) as compared to other concentrations. Third region spans within -13.2 to $-11.8 \mathrm{eV}$ (energy range), represents major contribution of $\mathrm{Cd} d, \mathrm{~S} s$ and $\mathrm{Ag} d$ states with least impact of $\mathrm{Ag} p, \mathrm{Cd} s$ and $\mathrm{S} p$ states. It is clearly observed that in third region tip of $\mathrm{Ag} d$ states rises from first $(3.12 \%)$ to third $(9.37 \%)$ concentration but again start decreasing in last concentration $(12.5 \%)$. The reason may be associated with fact that dopant $(\mathrm{Ag})$ injection into host rock-salt CdS lattice can margin impurity states of conduction band. It may also be due to involvement of different valence electrons of dopant $\left(\mathrm{Ag}^{+}\right)$with that of $\mathrm{Cd}^{2+}$, causing bonding effect. Moreover, arrangement of extranuclear electrons is also different due to impurity states [32]. In all concentrations, it is important to note that $\mathrm{Ag} s$ and $p$-states are almost diminishing over lower energies but become significant over large values of energy. On other side, conduction band minimum contains $\mathrm{Cd} s, \mathrm{~S} p$ and $\mathrm{Ag} s$ states along with least population of $\mathrm{Cd} d, \mathrm{~S} s$ and $\mathrm{Ag} d$ states. Impurity level has localized states in vicinity of the Fermi level and bends toward conduction band. By increasing $\mathrm{Ag}$ concentration in host material, we observe leaning of impurity levels toward conduction band which shows that electro-conductivity has increased. Moreover, distribution of different states in three energy regions may be responsible for tuning optical and electronic properties with good conductivity of Ag substituted CdS system. Our PDOS results match with study reported by Jianbo Yin et al. in 2016 in which they calculated 

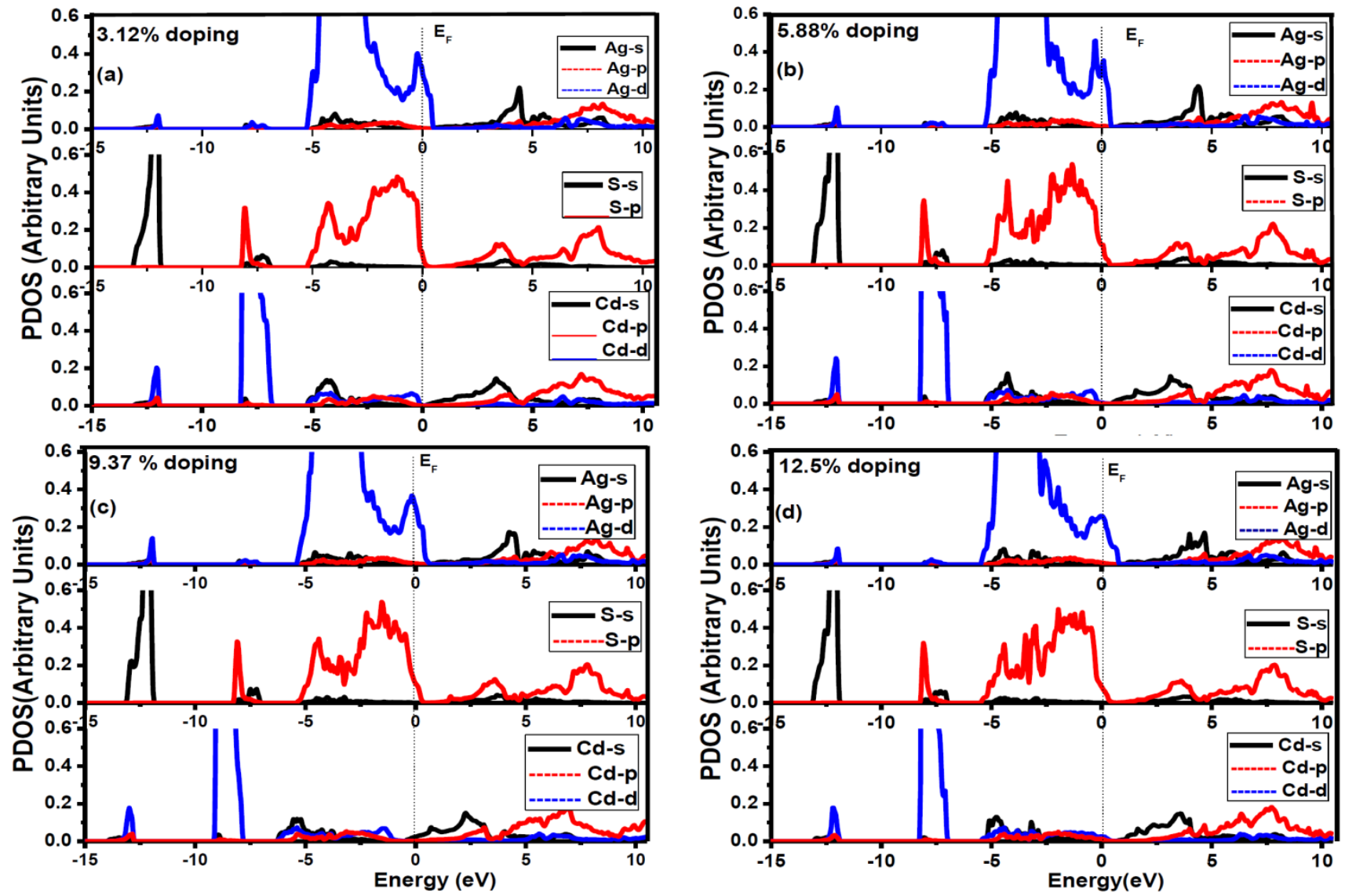

Fig. 3. PDOS for Ag doped CdS in case of (a) 3.12\%, (b) 5.88\%, (c) 9.37\%, and (d) $12.5 \%$ Ag-concentration effect.

photoluminescence and density of states of wurtzite CdS [32]. The difference may be due to assumption of rock-salt structure and DFT approximations. However, leaning of impurity states can be described by the expression,

$$
n=N_{c} \exp \left(-\frac{E_{c}-E_{\mathrm{F}}}{k_{\mathrm{B}} T}\right)
$$

where $N_{c}$ is the density of states, $n$ is concentration of electrons, $E_{c}$ is energy of conduction band edge, $k_{\mathrm{B}}$ is the Boltzmann constant and $T$ is absolute temperature [34]. Change in the concentration of electrons depends on the difference between $E_{c}$ and $E_{\mathrm{F}}$ in the equilibrium condition, which causes leaning of impurity states toward conduction band. Therefore, extrinsic semiconductor shows an imbalance in the electron and hole concentration because the dopants are fully ionized. Due to this, the Fermi level shifts from the center of the band gap toward either the conduction band or valence band depending on the type of dopant. That is why the dopant injection into host CdS lattice can induce impurity states in the band gap region due to involvement of different valence electronic states of $\left(\mathrm{Ag}^{+}\right)$with that of $\mathrm{Cd}^{2+}$.

Plots of TDOS represent curves which are obtained as solution of the Kohn-Sham equations. Occupied and unoccupied energy levels have been plotted in energy range -15 to $10.5 \mathrm{eV}$. Different graphical trends with various concentrations can be seen in Fig. 4 which is an interpretation for TDOS. After quick analysis of TDOS plots for all concentrations it is interpreted that role of dopant atom $(\mathrm{Ag})$ is vital in entire story of electron transition from valence to conduction band. Hence it may provide sufficient number of electrons necessary for enhancing conductivity of $\mathrm{CdS}$ :Ag material. TDOS plots contain states distribution of individual atoms in narrow and spacious regions. Due to the addition of $\mathrm{Ag}$, impurity states are created which cross over the Fermi level extending themselves into the conduction band region. In valence band, first region is occupied within -5.4 to $-0.3 \mathrm{eV}$ energy and shows coupling of states where $\mathrm{Ag}$ states are most prominent with least contribution of $\mathrm{Cd}$. First region in case of $\mathrm{CdS}: \mathrm{Ag}(9.33 \%)$ is extending towards energy range from -1 to $-6.3 \mathrm{eV}$. Second region spread over -6.8 to $-8.3 \mathrm{eV}$ for pure CdS now shifted toward lower energies due to doping of $\mathrm{Ag}$, which occupies maximum energy states of $\mathrm{Cd}$ atom with minimum role of $\mathrm{Ag}$ atom. However, a large shift appears for CdS:Ag $(9.33 \%)$ bounded between -9.2 to $-7.7 \mathrm{eV}$ but coupling of states in this region is same as rest of concentrations. Third region situated between -13.0 to $-11.5 \mathrm{eV}$ for pure CdS is now shifted to -13.2 to $-11.8 \mathrm{eV}$ by doping of $\mathrm{Ag}$, in which sulfur states are dominant with minimum contribution of $\mathrm{Ag}$ states. A bit different behavior 

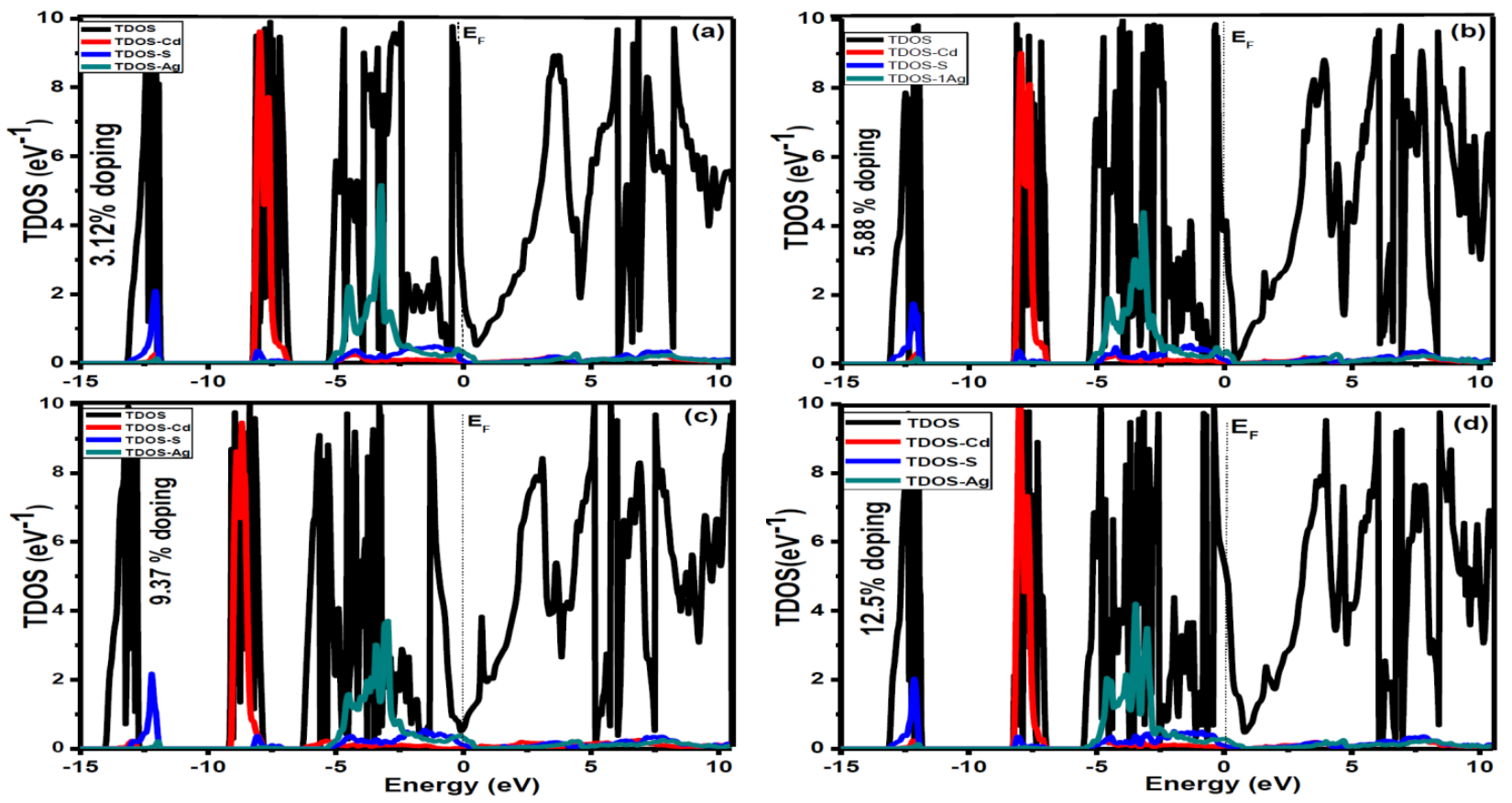

Fig. 4. TDOS of Ag doped CdS in case of (a) 3.12\%, (b) 5.88\%, (c) 9.37\%, and (d) $12.5 \%$ Ag-concentration effect.

is traced in third concentration where third region in valence band spans remarkably wide over interval of -14.1 to $-12.8 \mathrm{eV}$ as evident from Figs. 2 and 4 . Hence, effect of impurity $(\mathrm{Ag})$ addition causes generation of more states which plays significant role in optical and electronic properties. Furthermore, it is also illustrated by Fig. 4, that dopants states in the valence band decrease in vicinity of the Fermi level from $5 \mathrm{eV}^{-1}$ for least dopant concentration to below $4 \mathrm{eV}^{-1}$ up to third concentration $(9.37 \%)$ but for $\mathrm{Ag}$ concentration of $12.5 \%$, it increases again above $4 \mathrm{eV}^{-1}$ causing a favorable change in optical and electronic properties.

Through TDOS analysis, it is admiring to mention that as Ag concentration is increased, more states transferred to conduction band which in result increases conductivity and optical properties are enhanced. Since dopant states cross the Fermi level, its role becomes dominant upon increasing its concentration which emphasize its importance for many applications. Due to this reason, Ag substituted CdS system is most suitable for devices used in optoelectronic and spintronic.

\subsection{Optical properties}

Optical properties are important due to fact that they help in transforming technology at nanoscale. Research on $\mathrm{CdS}$ nanoparticles becomes significant because of quantum confinement effects which predict optical properties. An unprecedented change in structural, optical, and electrical properties may emerge due to doping with certain metals in CdS host lattice. Ag substituted CdS system may highlight quantum trap for excited electrons which in turn helps in understanding basic physics, encoding possible changes in physical properties. CdS:Ag doped system exhibits nominal sensitivity while studying absorption effects which appear due to interaction of electromagnetic radiations. Results presented in current study, are obtained by DFT calculations and plots (Figs. 5-7) for optical absorption, conductivity, extinction coefficient, reflectivity, index of refraction and dielectric constant are sketched. Graphical trends are analogous to existing literature findings. Figure 5 a represents optical absorption for various concentrations including pure CdS. Evidently, absorption increases with increase in energy up to certain energy value leading to absorption maxima, which afterwards shows decreasing trend. Absorption trends for pure $\mathrm{CdS}$ and various Ag concentrations have been shown in energy range 0-8 eV. Absorption of pure $\mathrm{CdS}$ is maximum at $4.8 \mathrm{eV}$ while for all $\mathrm{Ag}$ concentrations peaks lie at different energies than that of pure CdS which shows change in optical properties after Ag doping. Graphical trends for all concentrations are almost similar but for highest Ag-concentration (12.5\%), behavior is relatively different illustrating peak splitting, and having maximum absorption at $5.0 \mathrm{eV}$. From graphical manifestation, it can be seen that all graphs are gradually increasing and then decreasing after attaining maximum values to certain $5.9 \mathrm{eV}$. It then increases further up to $6.4 \mathrm{eV}$ for all concentrations and decreases afterwards. Absorption trend decreases within 5-8 eV. Beyond maximum value of absorption for all concentrations, oscillatory trend is observed which is almost constant for higher energy values. This increasing trend of absorption matches with the results presented by Junaid Iqbal Khan et al. [20] where they studied optical properties of $\mathrm{Cu}$ doped rock-salt $\mathrm{CdS}$ system. It is further noted that absorption curves shift slightly towards higher energies 

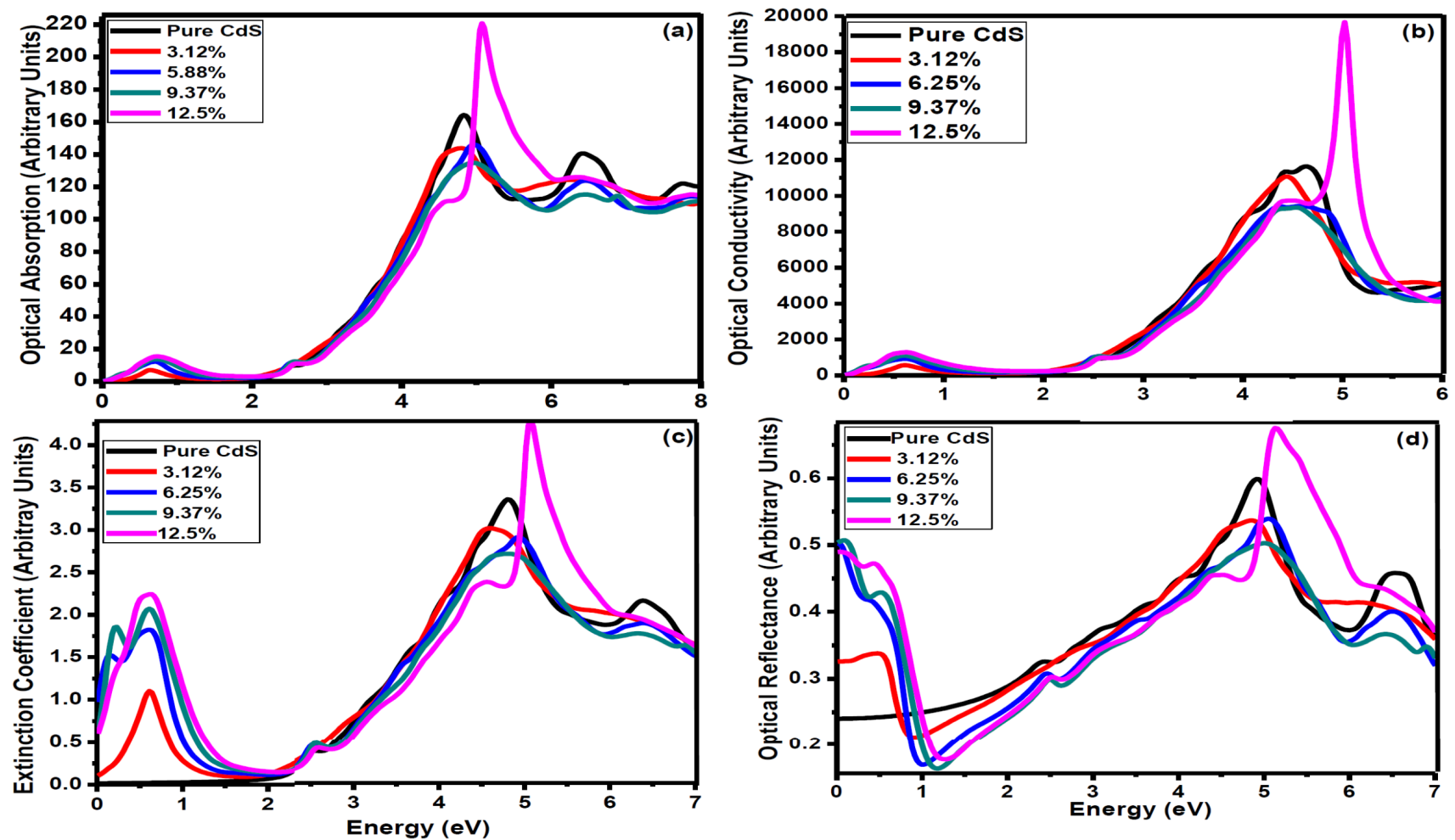

Fig. 5. Plots of (a) optical absorptions, (b) optical conductivity, (c) extinction coefficient, (d) optical reflectance of Ag doped CdS system.

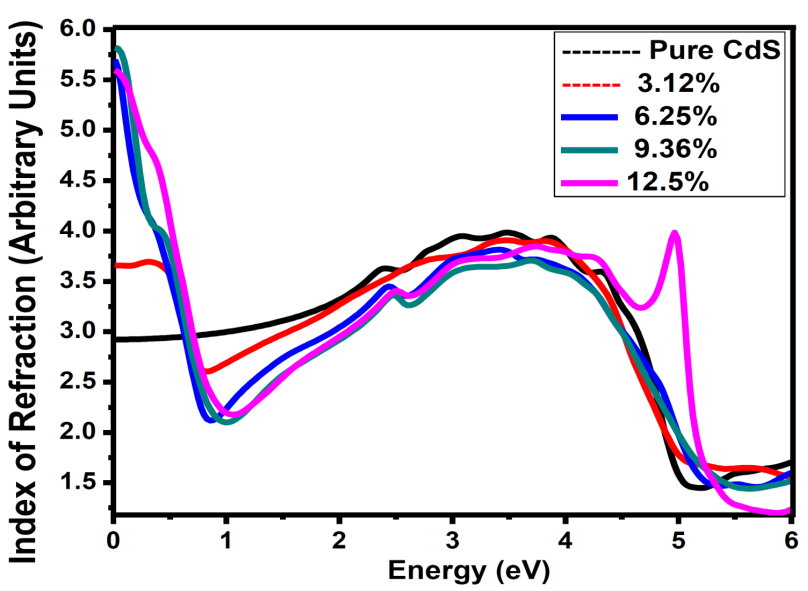

Fig. 6. Index of refraction of (a) pure $\mathrm{CdS}$ and $\mathrm{Cd}: \mathrm{Ag}$ system with (b) $3.12 \%$, (c) $6.25 \%$, (d) $12.5 \%$ Agconcentration effect.

and the absorption maxima moves from $4.8 \mathrm{eV}$ for pure $\mathrm{CdS}$ to $5.08 \mathrm{eV}$ for $\mathrm{CdS}$ with $12.5 \% \mathrm{Ag}$ as depicted by Fig. 5a. Therefore, it is reasonable to state that by increasing Ag concentration, absorption spectrum is blue shifted upon increase in photon energy (frequency). Shift in absorption peaks may be related to quantum size effect and is associated with decrease in crystallite size [35]. Shift in absorption peaks with increase of Ag concentrations represents blue shift which points strong bonding and interaction of dopant $(\mathrm{Ag})$ atoms with $\mathrm{CdS}$ [36]. Blue shift may be associated with reasoning that introducing
$\mathrm{Ag}$ into host rock-salt CdS lattice induces defects, which cause shift in absorption curves. However, change in electronic transitions brings change in absorption curves blue shift [32]. This blue shift may be associated with reasoning that CdS:Ag material is in nanoscale regime as discussed by Muruganandam [37]. Blue shift explains nanoapplications of $\mathrm{Ag}$ doped $\mathrm{CdS}$ which can be used commercially in device fabrication. Silver doped CdS system exhibits high absorption in ultraviolet region $(\approx 248 \mathrm{~nm})$; however, it decreases in visible and near IR regions. This blue shift in absorption spectrum is analogous to the results reported by Nithya and Boopathi [36] where they carried out experimental study of $\mathrm{Ag}$ doping effects on CdS with various concentrations and presented structural (XRD, SEM) and optical properties (absorption, FTIR) of CdS nanoparticles. Difference may be due to fact that they focused on experimental study of wurtzite CdS while our findings are theoretical, based on rock-salt CdS structure, calculated within DFT framework with certain approximation in Wien2k code. Blue shift appears due to introduction of $\mathrm{Ag}$ in host lattice (CdS) and may lead to structural defects as stated in [32]. Moreover, different peaks shift toward lower wavelength region may be connected to strong quantum confinement effects which are incumbent for enhancing optoelectronic properties of materials [38]. However, peak which emerges between 0 and $1.5 \mathrm{eV}$ may be associated with lattice defects due to ionic rearrangement because $\mathrm{Ag}^{+}$have different chemical valence than $\mathrm{Cd}^{2+}$. This fact might cause reordering 
of extranuclear electrons suggesting weak interaction of Ag to CdS [32]. Because of this, no plasmonic effects or appreciable broadening is observed [39]. So a high concentration of $\mathrm{Ag}$ is suggested because it will bring more prominent effects as obvious from $12.5 \%$ concentration in comparison to rest in our theoretical study. It is remarkable to mention that we do not observe any such peak in case of absorption of pure CdS spectrum. Higher absorption peaks with increase of $\mathrm{Ag}$ concentration is in accordance with the work reported by Salmana et al. in [40] while differences are due to theoretical formulations carried out with certain approximations and their work is based on experimental formulation.

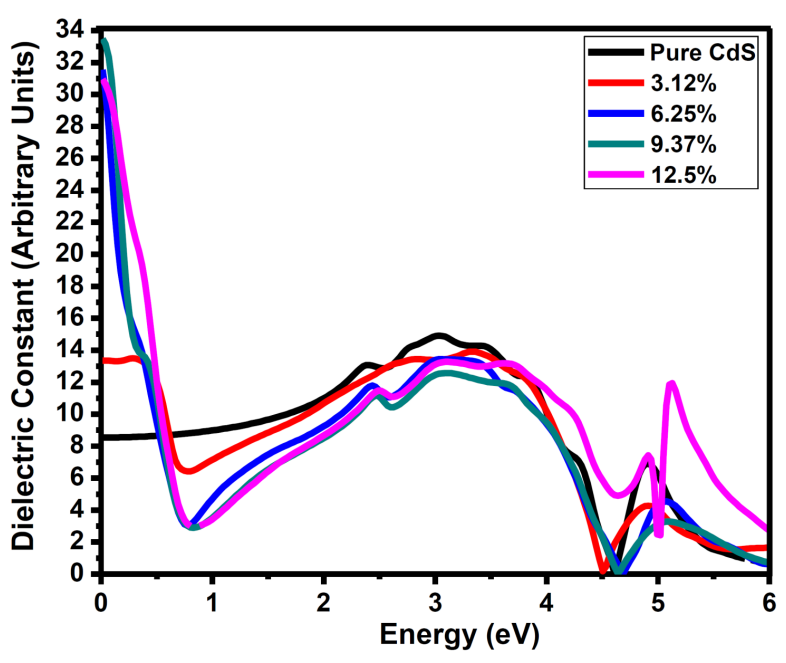

Fig. 7. Dielectric constant of (a) pure CdS and Cd:Ag system with (b) $3.12 \%$, (c) $6.25 \%$, (d) $12.5 \% \mathrm{Ag}$ concentration effect.

Graphs portrayed in Fig. 5b represent optical conductivity behavior of pure and Ag substituted CdS system for various concentrations. It can be seen that conductivity increases with increase in energy $(\mathrm{eV})$. Graph has been sketched between energy values 0 and $6 \mathrm{eV}$ along $x$-axis. Graphical trends are almost similar for pure and all concentrations except highest $(12.5 \%)$ concentration which has maximum conductivity at $5.02 \mathrm{eV}$. Conductivity curves are rising in all cases up to a certain value and then decrease which further become almost constant for higher energy values. It may be due to fact that during absorption more photonic energy is absorbed which excites electron transitions. These transitions of electrons in turn increase conductivity of CdS: $\mathrm{Ag}$ material. This reasoning may be associated with theoretical findings reported by Yan-Xiao Han et al. in [41].

In optics, extinction coefficient is very important and physically describes absorbance of radiations for a particular wavelength. It is complex part of refractive index and is related to light absorption. Figure $5 \mathrm{c}$ represents change in plots of extinction coefficient for pure and silver doped CdS for various concentrations. The behavior of graph is marked with energy variation in the range
0-7 eV along $x$-axis. Plots are obtained by DFT calculations simulated for many electrons system. From Fig. 5c, it can be seen that plots of extinction coefficient are increasing with increase in photon energy. Extinction coefficients are related to absorption coefficient by relation

$$
k=\frac{\lambda \alpha}{4 \pi},
$$

where $k$ and $\alpha$ are extinction and absorption coefficients, respectively. Hence, we observe a clear resemblance between Fig. 5a and c. Constant increase and decrease in curves may be referred to absorption of light incident at grain boundaries [42]. Increase in extinction coefficient values with corresponding energies may be reasoned due to the quantum confinement effects which further connected to crystalline size and its variation [43]. Peaks which emerge in low energy region $0-1.5 \mathrm{eV}$ may be reasoned to incorporation of $\mathrm{Ag}^{+}$in host CdS lattice and also due to quantum effects which appeared during substitutions. The peaks may be associated to localized density of states near the Fermi edge upon addition of doping atom $(\mathrm{Ag})$. It is clearly remarked that extinction coefficient curves are more pronounced in case of highest concentration (12.5\%). However, DFT values for $k$ are larger $(0.6-1.0)$ than experimental value $(k=0.5)$ for pure CdS thin films at energy $1.96 \mathrm{eV}$ (632.8 nm) [44]. All concentrations excluding $3.12 \%$ concentration (for which $k=0.1$ ) have higher extinction coefficient values. These trends for extinction coefficient have good agreement with experimental work reported in [40].

Figure 5d represents graph of optical reflectivity for pure and $\mathrm{Ag}$ doped $\mathrm{CdS}$ with various concentrations as simulated by DFT. Various curves are obtained while plotting reflectivity with energy variation between 0 and $7 \mathrm{eV}$ along $x$-axis. Graph for pure CdS shows steady increase which to an extent resembles to that of $3.12 \%$ $\mathrm{Ag}$ concentration but for rest of concentrations different trends are observed. However, for all concentrations of $\mathrm{Ag}$ doping, reflectivity first decreases rapidly up to $1.2 \mathrm{eV}$ and then increases gradually for further rise of photon energy. It can also be seen from Fig. $5 \mathrm{~d}$ that the reflectivity of $6.25 \%, 9.37 \%$ and $12.5 \%$ first decreases in different energy regions. It then shows steady rise in certain steps until $3.12 \%(4.8 \mathrm{eV}), 6.25 \%(5.0 \mathrm{eV}), 9.37 \%(5.0 \mathrm{eV})$ and $12.5 \%(5.1 \mathrm{eV})$. It further decreases suddenly, increases and then becomes almost constant for high energy values. Reflectivity increases in visible region as we increase $\mathrm{Ag}$ content in CdS lattice. The unusual trend of reflectivity of $\mathrm{Ag}$ doped $\mathrm{CdS}$ system in energy range 0-1.5 eV may be due to incorporation of $\mathrm{Ag}^{+}$in host CdS lattice. Reflectivity trends matches with the work as reported in [40, 45] but difference may be due to reason that their calculations are experimental based while our calculations are theoretical encoded within DFT simulation.

For better comprehension of optical properties of any system, refractive index is very important physical quantity which needs to be analyzed. It helps in understanding atomic interactions at microscopic level. In Fig. 6, a graphical analysis of refractive index is presented. Graph 
is meant for $\mathrm{Ag}$ doped $\mathrm{CdS}$ for various concentrations including pure CdS. Variations in curves for all concentrations have been observed in energy $0-6 \mathrm{eV}$ along $x$ axis. It can be seen that graph of $3.12 \%$ concentration increases slightly and decreases $(0.7 \mathrm{eV})$ while for rest of higher concentration, graph expresses sudden decrease $6.25 \%(0.8 \mathrm{eV}), 9.36 \%(0.9 \mathrm{eV})$, and $12.5 \%(1.0 \mathrm{eV})$. After this, graph for all concentrations shows slight increase in different steps until it attains maximum value at $3.12 \%$ $(3.8 \mathrm{eV}), 6.25(3.4 \mathrm{eV}), 9.36(3.6 \mathrm{eV})$, and $12.5 \%(4.96 \mathrm{eV})$. The graph then decreases and adopts constant behavior for higher energy values. Sharp increase in value of index of refraction within energy 1.0 to $4.9 \mathrm{eV}$, represents energy losses which may occur due to scattering, free carrier absorption, photogeneration and phonon generation [42]. Index of refraction has highest value $\approx 5.79$ for third concentration $(9.36 \%)$. For other concentrations, refractive indices are $3.12 \% \approx 3.6,6.25 \% \approx 5.6$, and $12.5 \% \approx 5.59$. Experimental calculated value of refractive index is 2.38 at energy $1.96 \mathrm{eV}(632.8 \mathrm{~nm})$ while our DFT calculated values are higher than experimental value. Hence high value of refractive index of $\mathrm{Ag}$ doped CdS system emphasizes its use in optoelectronic devices [31, 46, 47].

Photon has electric field which is responsible for optical transition of occupied and unoccupied states. Because of this reason, optical properties of particularly dielectric constant and extinction coefficient carry mandate while focusing on optics of any physical system. Dielectric function consists of real and imaginary parts

$$
\epsilon=\epsilon_{1}+\mathrm{i} \epsilon_{2},
$$

where $\left(\epsilon_{1}, \epsilon_{2}\right)$ are real and imaginary parts of dielectric constant, respectively. Real part $\left(\epsilon_{1}\right)$ is connected to anomalous behavior and polarization while imaginary part is defined in terms of real part of conductivity $\sigma$ (i.e. $\left.\epsilon_{2}=\frac{4 \pi}{\omega} \sigma\right)$. This imaginary part represents energy dissipation into the material which might appear as conductivity and optical conductivity traits have already been expressed in Fig. 4b $[48,49]$. Figure 7 is a plot for dielectric constant of Ag doped CdS system with various concentrations effects. From cursory inspection of graph, it is evident that curve for pure CdS has steady increase but for $3.12 \%$ concentration increases up to energy $\approx 0.4 \mathrm{eV}$ and later on decreases suddenly. From Fig. 7, the dielectric constant depicts a sudden decrease in different energy regions in the vicinity of $0.8 \mathrm{eV}$ for all $3.12 \%, 6.25 \%, 9.37 \%$, and $12.5 \%$ concentrations. After the minima, a steady increase in trend is traced in between 0.8 and $3.0 \mathrm{eV}$ which suddenly decreases again to $4.6 \mathrm{eV}$ but the curves have been displaced little bit for all concentrations. Another peak emerges at $4.9 \mathrm{eV}$ and afterward graph becomes constant for high energy values. However, static dielectric constant values for various concentrations are 8.6 (pure CdS), 13.27 (3.12\%), $31.80(6.25 \%), 33.64(9.37 \%)$, and $30.70(12.5 \%)$. These values are higher than experimental value of CdS [20,50]. Results are in good agreement with the findings reported in $[31,40]$. Most of dielectric properties appear in low energy (frequency) regions due to quantum confinement effects. Moreover, theoretical DFT based calculations exhibit dependence of high energy dielectric constant on valence electron plasmon energy.

\section{Conclusion}

We studied PDOS, TDOS and optical properties of Ag doped CdS using Wien2k code for DFT calculations by applying PBE-GGA approximation. Optical absorption including conductivity, extinction coefficient, optical reflectance, index of refraction, and dielectric constant for varying concentrations of dopant $(\mathrm{Ag})$ with fixed supercell size $(1 \times 2 \times 2)$ show slight change due to quantum effects, lattice defects, and Ag substitutions. Blue shift in absorption spectrum and a decrease in dielectric constant are observed as we increase doping concentration. Optical conductivity is enhanced upon doping $\mathrm{Ag}$ into $\mathrm{CdS}$ and most contributing orbitals are $\mathrm{Cd} s, \mathrm{~S} p$ and $\mathrm{Ag} d$.

\section{Acknowledgments}

This work is carried out in Department of Physics, Bahauddin Zakariya University, Multan and authors would like to acknowledge the utilization of special computational facilities of Department of Physics, School of Science and Engineering, Lahore University of Management Sciences (LUMS), Lahore, Pakistan.

\section{References}

[1] U. Pal, R. Silva-González, G. Martínez-Montes, M. Gracia-Jiménez, M.A. Vidal, Sh. Torres, Thin Solid Films 305, 345 (1997).

[2] J. Kulkarni, O. Kazakova, J. Holmes, Appl. Phys. A 85, 277 (2006).

[3] S. Chandramohan, A. Kanjilal, S.N. Sarangi, S. Majumder, R. Sathyamoorthy, C.-H. Hong, T. Som, Nanoscale 2, 1155 (2010).

[4] Sunil Kumar, Zinki Jindal, Nitu Kumari, Narendra Kumar Verma, J. Nanopart. Res. 13, 5465 (2011).

[5] J. Wang, M. Isshiki, 10.1007/978-0-387-29185-7 16 in:Springer Handbook of Electronic and Photonic $\bar{M} a-$ terials Eds. S. Kasap, P. Capper, Springer, Boston (MA) 2006, p. 325.

[6] I. Gur, N.A. Fromer, M.L. Geier, A.P. Alivisatos, Science 310, 462 (2005).

[7] H. Uda, S. Ikegami, H. Sonomura, Jpn. J. Appl. Phys. 29, 30 (1990).

[8] P.K. Santra, P.V. Kamat, J. Am. Chem. Soc. 134, 2508 (2012)

[9] Ying Guo, He Zhang, Yao Wang, Zuo-Lei Liao, GuoDong Li, Jie-Sheng Chen, J. Phys. Chem. B 109, 21602 (2005).

[10] M. Sadeghi, W. Liu, T.-G. Zhang, P. Stavropoulos, B. Levy, J. Phys. Chem. 100, 19466 (1996).

[11] K. Deng, L. Li, Adv. Mater. 26, 2619 (2014).

[12] H. Dedong, L. Ying-Kai, D.-P. Yu, Nanoscale Res. Lett. 10, 285 (2015). 
[13] X. Michalet, F.F. Pinaud, L.A. Bentolila, J.M. Tsay, S. Doose, J.J. Li, G. Sundaresan, A.M. Wu, S.S. Gambhir, S. Weiss, Science 307, 538 (2005).

[14] H. Du, G.Q. Xu, W.S. Chin, Chem. Mater. 14, 4473 (2002).

[15] M.A. Hossain, Z.Y. Koh, Q. Wang, Phys. Chem. Chem. Phys. 14, 7367 (2012)

[16] G. Brusatin, M. Guglielmi, P. Innocenzi, A. Martucci, G. Scarinci, J. Electroceram. 4, 151 (2000).

[17] C. Ferekides, D. Marinskiy, D. Morel, in: Conference Record of the Twenty Sixth IEEE Photovoltaic Specialists Conf., 1997, IEEE, Anaheim (CA) 1997, p. 339.

[18] Y.K. Liua, J.A. Zapien, C.Y. Geng, Y.Y. Shan, C.S. Lee, Appl. Phys. Lett. 85, 3241 (2004).

[19] S.G. Hickey, D.J. Riley, J. Phys. Chem. B 103, 4599 (1999).

[20] M. Junaid Iqbal Khan, Shahzeen Babar, Azeem Nabi, A. Manzoor Rana, Mazhar Iqbal, Saif Ur Rehman, Javed Ahmad, J. Alloys Comp. 695, 888 (2017).

[21] S.A. Masti, A.G. Godghate, A.S. Patil, M.M. Jadhav, Archiv. Phys. Res. 5, 51 (2014).

[22] Xiaoping Zou, Sheng He, Gongqing Teng, Chuan Zhao, J. Nanomater. 2014, 50 (2014).

[23] S. Etemadfard, M. Ghazi, M. Ehsani, Chalcogenide Lett. 8, 411 (2011).

[24] F. Atay, S. Kose, V. Bilgin, I. Akyuz, Mater. Lett. 57, 3461 (2003).

[25] V.I. Kozlovsky, V.A. Akimov, M.P. Frolov, Yu.V. Korostelin, A.I. Landman, V.P. Martovitsky, V.V. Mislavskii, Yu.P. Podmar'kov, Ya.K. Skasyrsky, A.A. Voron, Phys. Status Solidi B 247, 1553 (2010).

[26] P. Kumar, N. Saxena, A. Agarwal, V. Gupta, AIP Conf. Proc. 1661, 080017 (2015).

[27] N.A. Shah, A. Nazir, W. Mahmood, W.A.A. Syed, S. Butt, Z. Ali, A. Maqsood, J. Alloys Comp. 512 , 27 (2012).

[28] M. Ristova, M. Ristov, P. Tosev, M. Mitreski, Thin Solid Films 315, 301 (1998).

[29] D. Saikia, P.K. Saikia, P.K. Gogoi, P. Saikia, Dig. J. Nanomater Bios. 6, 589 (2011).

[30] G.Ya. Grodzyuk, V.V. Shvalagin, S.R. Lavorik V.M. Granchak, Theor. Exp. Chem. 50, 212 (2014).
[31] P.K. Mochahari, F. Singh, K.C. Sarma, J. Mater. Sci: Mater Electron. 29, 582 (2017).

[32] J. Yin, X. Lu, Q. Dong, J. Alloys Comp. 695, 1301 (2017).

[33] S.S. Boxi, S. Paria, RSC Adv. 4, 37752 (2014).

[34] B. Van Zeghbroeck, Principles of Electronic Devices, University of Colorado, Boulder (CO) 2011.

[35] M. Junaid Iqbal khan, Zarfishan Kanwal, M. Nauman Usmani, Mater. Res. Express. 5, 015915 (2018).

[36] N. Nithya, G. Boopathi, IJSR.NET 4, 2299 (2015).

[37] S. Muruganandam, G. Anbalagan, G. Murugadoss, Optik 131, 826 (2017).

[38] R. Chauhan, A. Kumar, R.P. Chaudhary, Res. Chem. Intermed. 39, 645 (2013).

[39] V.S. Taur, R.A. Joshi, R. Sharma, Int. J. Photoenergy 2012, 264027 (2012).

[40] Z.N. Salmana, F.A.M., Riydh, N. Ali, IJSBAR 21 149 (2015).

[41] Yan-Xiao Han, Chuan-Lu Yang, Yong-Tai Sun, MeiShan Wang, Xiao-Guang Ma, J. Alloys Comp. 585. 503 (2014).

[42] M. Kamruzzaman, T. Luna, J. Podder, Innovat. Syst. Des. Eng. 2, 117 (2011).

[43] L. Soliman, H. Afify, I. Battisha, Indian J. Pure Appl. Phys. 42, 12 (2004).

[44] Filmetrics, Refractive Index of CdS, Cadmium Sulfide, 2016.

[45] R. Ganesh, V. Senthil Kumar, K. Panneerselvam, M. Raja, Arch. Phys. Res. 4, 97 (2013).

[46] S. Thirumavalavan, K. Mani, S. Sagadevan, J. Ovonic Res. 11, 203 (2015)

[47] T.K. Chaudhuri, M.G. Patel, AIP Conf. Proc. 1313 , 275 (2010).

[48] E. Yaldiz, M. Bayrak, Math. Comput. Appl. 8, 303 (2003).

[49] M. Dresselhaus, M. Dresselhaus, in: Proc. Int. School of Physics "Enrico Fermi", Ed. J. Tauc, Academic Press, New York 1966, p. 198.

[50] Ping Li, Chang-Wen Zhang, Jie Lian, Shang Gao, Xiao Wang, Solid State Commun. 151, 1712 (2011). 